\title{
Quantum Mechanics on the cylinder
}

\author{
José A. Gonzálezł, Mariano A del Olmo§ and Jaromir Tosiek\| \\ Departamento de Física Teórica, Universidad de Valladolid \\ 47011 Valladolid, Spain
}

\begin{abstract}
A new approach to deformation quantization on the cylinder considered as phase space is presented. The method is based on the standard Moyal formalism for $\mathbb{R}^{2}$ adapted to $S^{1} \times \mathbb{R}$ by the Weil-Brezin-Zak transformation. The results are compared with other solutions of this problem presented by Kasperkovitz and Peev [Ann. Phys. 230, 21 (1994)] and by Plebański and collaborators [Acta Phys. Pol. B 31, 561 (2000)]. The equivalence of these three methods is proved.
\end{abstract}

To be published in J. Opt. B: Quantum Semiclass. Opt. (2003).

PACS numbers: 03.65.Sq, 02.30.Uu

$\ddagger$ E-mail: jagonzal@fta.uva.es

$\S$ E-mail: olmo@fta.uva.es

|| E-mail: tosiek@ck-sg.p.lodz.pl

I On leave of absent from Technical University of Lodz (Poland). 


\section{Introduction}

Mathematical formalisms of classical and quantum physics are completely different. In the first one physical systems are described on differential manifolds, in the second one linear operators and vectors on Hilbert spaces are the suitable objects to describe quantum systems. However, there are formal analogies among them, especially between quantum mechanics and classical statistical mechanics. Thus, since the beginning of quantum mechanics the question appeared if states and observables could be expressed in terms of functions on some phase space, as it happens in classical theory. Such a formalism would be more intuitive and would enlighten the relation between classical and quantum mechanics.

Moyal [1] basing on previous works by Weyl [2], Wigner [3] and Groenewold [4] presented quantum mechanics as a statistical theory (see also [5, 6, 7]). Unfortunately, his method can be applied only to systems with Euclidean configuration space. Quantum states are represented by normalized functions on a phase space (Wigner functions) but these functions cannot be interpreted as probability density since they are not always positive. The domains of Wigner functions are low bounded on the contrary to the classical theories (for instance, the distribution $\delta\left(q-q^{\prime}\right) \delta\left(p-p^{\prime}\right)$ that corresponds to a classical particle in $\mathbb{R}^{2}$ cannot be a Wigner function). These facts reflect the Heisenberg uncertainty principle.

With respect to observables, they are represented by real functions on phase space. However, their classical commutative product has to be substituted by a noncommutative multiplication (*-product). The Moyal bracket (the analog of the commutator of operators), which characterizes the Lie algebra of observables, is different, in general, from the classical Poisson bracket.

On the other hand, quantum mechanics formalism on phase space can be defined in an independent way, without any explicit mention to the Weyl correspondence, like an algebra of functions defined on phase space with the Moyal product as fundamental element. This was the approach followed by Berezin $[8,9]$ in his quantization method. The development of this idea carried to a new theory: deformation quantization [10], where the quantum formalism is obtained by deforming the algebra of classical observables by the introduction of a new noncommutative product ( $*$-product) between the functions on phase space. In some sense, this theory represents respect to Moyal's formalism an analog role to the geometric quantization (see for instance [11, 12] and references therein) respect to the standard formalism. Berezin's method can be seen as a particular case of deformation quantization valid for kählerian varieties, which permits to use coherent states to obtain the $*$-product [13].

In this paper we pretend to develop a quantum formalism for physical systems with non-euclidean phase spaces. We present the particular case of the cylinder as phase space with nontrivial topology. Our approach is based on the well known formalism on $\mathbb{R}^{2}$, in particular on the Weyl correspondence in $\mathbb{R}^{2}$ that it is carried to the cylinder using the Weil-Brezin-Zak transform [14, 15, 16]. 
Since to quantize means to establish a bijective correspondence between classical and quantum observables, in principle it will be possible in any case to invert it and to obtain a new correspondence assigning a function (symbol) on the phase space to each observable or quantum state. In this case the correspondence will be used to construct a quantum formalism in phase space without making use of the canonical quantization. So, quantization and phase space formalism revel as two aspects of a same problem and have to be studied simultaneously.

In the literature there exist several quantum formalisms on phase spaces related with the presented here. For instance, Berezin studied the cylinder quantization in [8] showing that the "admisible" manifold is a set of paralell circles, such that the distance between two consecutive circles is $\hbar / 2$. Berry [17], in a work about the quantum rotor, and Mukunda [18], in a study about angle variables, developed independently the same formalism. Since their results gave a very complicated expression for the *-product, Kasperkovitz et al. [19] introduced a modified formalism which describes the motion of two particles on the circle. It was used by Arnold et al. [20] to study the dynamics of Bloch electrons. Later, Kasperkovitz and Peev [21] completed all these ideas introducing some quantum formalisms on phase spaces for systems with some kind of periodicity. In particular, they considered systems where the position variable moves on a circle, which is the case studied here. In ref. [22, 23] Arratia et al. used the Moyal quantization method and profited the fact that the cylinder is a coadjoint orbit, to construct Moyal quantizers. More recently, Plebański and collaborators [24] constructed a quantization algorithm based on a family of unitary operators.

Thus, we can conclude that it seems necessary a more suitable framework to relate the quantum formalisms on the plane and on the cylinder. We show in this work that such a relation is easily realized by the so-called Weil-Brezin-Zak (WBZ) transform [26] which, under different forms, has been used in the past for the study of periodic potentials and other related problems [27]. We will see that the WBZ transform not only allows us to reproduce in a natural way all the known results for the quantum formalism on the cylinder, but also provides us with a solution to two important topics: coherent states and quantization. We will also prove in this work that Kasperkovitz and Peev's formalism as well as Plebański and collaborators formalism are equivalent to ours.

The paper is organized as follows. In section 2 we review the foundations of the quantizations via Weyl and Moyal approaches. In section 3 we quantize the cylinder as phase space using the Weil-Brezin-Zak transform that allows us to translate the well known Weyl formalism on $\mathbb{R}^{2}$. Section 4 contains a brief resume of the quantum formalism introduced in ref. [24]. Some conclusions and remarks close the paper. In particular, we prove the equivalence of our Moyal quantization procedure with the Kasperkovitz-Peev [21] and Plebański and collaborators [24] formalisms. 


\section{Moyal quantization}

\subsection{Weyl mappings}

One of fundamental obstacles which appear in a quantization procedure is establishing some one-to-one relation between classical and quantum observables. When the classical theory is formulated in terms of symplectic geometry and the quantum world is described in a Hilbert space, to quantize means to find some bijection between a set of functions and a collection of linear operators.

It is usually assumed that in the case of $\mathbb{R}^{2}$ (generalization for $\mathbb{R}^{2 n}$ is straightforward) operators $\widehat{Q}$ and $\widehat{P}$ representing canonical observables $q$ and $p$ respectively, are chosen like in the canonical quantization programme, i.e. they are self-adjoint and fulfill the commutation relation

$$
[\widehat{Q}, \widehat{P}]=i \hbar \widehat{1}
$$

Operators $\widehat{Q}$ and $\widehat{P}$ are unbounded, which originates problems about definition domains and self-adjoint extensions. These troubles disappear if unitary operators, obtained by exponentiation of $\widehat{Q}$ and $\widehat{P}$, are used. Weyl [2] proposed to substitute the canonical quantization rules for the postulate that the exponential function $e^{i(y q+x p)}, x, y \in \mathbb{R}$, has to be associated with the operator $e^{i(y \widehat{Q}+x \widehat{P})}$. In other words, whereas the canonical quantization determines some representation of the Heisenberg algebra by self-adjoint operators, the Weyl quantization gives a unitary representation of the Heisenberg group, equivalent up to a factor, to the Schrödinger representation.

It is well known that any tempered distribution $f$ can be written in terms of exponentials by means of its Fourier transform $\widetilde{f}$ on $\mathbb{R}^{2}$, namely

$$
f(q, p)=\frac{1}{2 \pi \hbar} \int_{-\infty}^{\infty} d y \int_{-\infty}^{\infty} d x \widetilde{f}(y, x) e^{i(y q+x p) / \hbar} .
$$

The Fourier transform is defined by

$$
\widetilde{f}(y, x)=\int_{-\infty}^{\infty} d q \int_{-\infty}^{\infty} d p f(q, p) e^{-i(y q+x p) / \hbar} .
$$

This expression suggests the following definition of the Weyl application

$$
\widehat{f}=\frac{1}{2 \pi \hbar} \int_{-\infty}^{\infty} d y \int_{-\infty}^{\infty} d x \widetilde{f}(y, x) e^{i(y \widehat{Q}+x \widehat{P}) / \hbar} .
$$

After some computations one gets that in coordinate representation

$$
(\widehat{f} \psi)(x)=\frac{1}{2 \pi \hbar} \int_{-\infty}^{\infty} d y \int_{-\infty}^{\infty} d p f\left(\frac{1}{2}(x+y), p\right) e^{i(x-y) p / \hbar} \psi(y)
$$

with $\psi \in \mathcal{S}(\mathbb{R})(\mathcal{S}(\mathbb{R})$ being the Schwartz space). So, $\widehat{f}$ is an integral operator with kernel

$$
K_{f}(x, y)=\frac{1}{2 \pi \hbar} \int_{-\infty}^{\infty} d p f\left(\frac{1}{2}(x+y), p\right) e^{i(x-y) p / \hbar}
$$

which acts as $L(\mathcal{H}): \mathcal{S}(\mathbb{R}) \rightarrow \mathcal{S}^{\prime}(\mathbb{R})\left(\mathcal{S}^{\prime}(\mathbb{R})\right.$ denotes the space of tempered distribution) [26, 28, 29]. 
From the last expression one can obtain that

$$
f(q, p)=\int_{-\infty}^{\infty} d y e^{i y p / \hbar} K_{f}\left(q-\frac{y}{2}, q+\frac{y}{2}\right) .
$$

So, the tempered distribution $f(q, p)$ is determined by the kernel.

Relation (2.5) is known as the Weyl correspondence. Formulas (2.3) and (2.5) are connected with Weyl ordering of operators. Generalization of them for other orderings can be found in [30].

\subsection{Moyal quantizer}

Weyl application rule (2.3) does not have a suitable form to extend it for systems with phase spaces different from $\mathbb{R}^{2 n}$, since it is associated with the concept of Fourier transform.

Let us come back to (2.4) and introduce a new variable $q=\frac{1}{2}(x+y)$. Now

$$
(\widehat{f} \psi)(x)=\frac{1}{\pi \hbar} \int_{-\infty}^{\infty} d q \int_{-\infty}^{\infty} d p f(q, p) e^{i 2 p(x-q) / \hbar} \psi(2 q-x) .
$$

Defining the family of operators parametrized by $q, p \in \mathbb{R}$

$$
(\widehat{\Omega}(q, p) \psi)(x):=\frac{1}{\pi \hbar} e^{i 2 p(x-q) / \hbar} \psi(2 q-x),
$$

we can rewrite the Weyl application form as

$$
\widehat{f}=\int_{R^{2}} f(q, p) \widehat{\Omega}(q, p) d q d p
$$

where

$$
\widehat{\Omega}(q, p):=\frac{1}{2 \pi \hbar} \int_{-\infty}^{\infty} d y \int_{-\infty}^{\infty} d x e^{-i(y q+x p) / \hbar} e^{i(y \widehat{Q}+x \widehat{P}) / \hbar} .
$$

It is easy to see that in (2.7) the term containing Fourier transformations has been

hidden in the symbol $\widehat{\Omega}(q, p)$. It suggests that in case of systems different from $\mathbb{R}^{2 n}$ to quantize them it is sufficient to define some family of operators $\widehat{\Omega}(q, p)$ and to substitute the integral over $\mathbb{R}^{2 n}$ by integration over the symplectic space $\Gamma$ of the system.

Operators $\widehat{\Omega}(q, p)$ were introduced independently by Grossmann [31] and Royer [32]. In the past they were called "Stratonovich-Weyl quantizers".

Let us analyse some basic properties of $\widehat{\Omega}(q, p)$ in $\mathbb{R}^{2}$.

Firstly, note that (see (2.6))

$$
(\widehat{\Omega}(0,0) \psi)(x)=\frac{1}{\pi \hbar} \psi(-x)
$$

represents (up to a factor) a reflection with respect to the origin. Hence, the operators $\widehat{\Omega}(q, p)$ can be seen as parity operators on phase spaces. Moreover, it is possible to prove that they are self-adjoint and the map $\mathbb{R}^{2} \rightarrow \widehat{\Omega}(q, p)$ is bijective. 
It has been showed [33] that

$$
\begin{aligned}
\operatorname{tr} \widehat{\Omega}(q, p) & =1, \quad \forall q, p \in \mathbb{R}, \\
\operatorname{tr}\left\{\widehat{\Omega}(q, p) \widehat{\Omega}\left(q^{\prime}, p^{\prime}\right)\right\} & =\delta\left(q-q^{\prime}\right) \delta\left(p-p^{\prime}\right),
\end{aligned}
$$

where both of expressions have to be seen in the sense of the distributions. The first expression of (2.9) is equivalent to the statement that the constant function equal to 1 is associated with the identity operator. The second property, called traciality, allows us to rewrite the formula for the Weyl correspondence as

$$
f(q, p)=\operatorname{tr}\{\widehat{f} \widehat{\Omega}(q, p)\} .
$$

Moreover, after some computations one obtains

$$
\operatorname{tr}\{\widehat{f} \widehat{g}\}=\int_{\mathbb{R}^{2}} f(q, p) g(q, p) d q d p .
$$

The Moyal product of two functions on phase space is defined by $\widehat{(f \star g)}:=\widehat{f} \widehat{g}$. It can be computed from (2.6) and (2.10) by the following formula

$$
(f \star g)(q, p)=\int_{\mathbb{R}^{2}} d q^{\prime} d p^{\prime} \int_{\mathbb{R}^{2}} d q^{\prime \prime} d p^{\prime \prime} \operatorname{tr}\left\{\widehat{\Omega}(q, p) \widehat{\Omega}\left(q^{\prime}, p^{\prime}\right) \widehat{\Omega}\left(q^{\prime \prime}, p^{\prime \prime}\right)\right\} f\left(q^{\prime}, p^{\prime}\right) g\left(q^{\prime \prime}, p^{\prime \prime}\right) .
$$

The equality holds

$$
\int_{\mathbb{R}^{2}}(f \star g)(q, p) d q d p=\int_{\mathbb{R}^{2}} f(q, p) g(q, p) d q d p .
$$

By analogy with the case $\mathbb{R}^{2}$ some axioms have been established to be fulfilled by operators $\widehat{\Omega}(q, p)$ in a general situation.

Let $\Gamma$ be an arbitrary phase space of a physical system, and $\mathcal{H}$ the corresponding Hilbert space of quantum states of this system.

The Moyal quantization is a map $\widehat{\Omega}: f \mapsto \widehat{f}$ among functions (or distributions) on $\Gamma$ and operators $L(\mathcal{H})$, given by

$$
\widehat{f}=\int_{\Gamma} f(u) \widehat{\Omega}(u) d u,
$$

where $d u$ is the volume determined by the symplectic form on $\Gamma$. The operator $\widehat{\Omega}$ is the Moyal quantizer defined as a map from $\Gamma$ in the set of operators $L(\mathcal{H})$, such that:

(i) $\widehat{\Omega}$ is bijective,

(ii) $\widehat{\Omega}(u)$ is self-adjoint for $\forall u \in \Gamma$,

(iii) $\operatorname{tr} \widehat{\Omega}(u)=1$ in the distributional sense for $\forall u \in \Gamma$,

(iv) Traciality:

$$
\int_{\Gamma} \operatorname{tr}\{\widehat{\Omega}(u) \widehat{\Omega}(v)\} \widehat{\Omega}(v) d v=\widehat{\Omega}(u)
$$

for $\forall u \in \Gamma$. 
For elementary physical systems with a symmetry group $G$ their phase spaces $\Gamma$ shall be, in general, coadjoint orbits (homogeneous spaces) of $G$ and $\mathcal{H}$ will carry a unitary irreducible representation $U$ of $G$ associated with $\Gamma$. The kernel $\widehat{\Omega}$ should verify an additional condition (covariance property)

$$
\widehat{\Omega}(g \cdot u)=U(g) \widehat{\Omega}(u) U\left(g^{-1}\right), \quad \forall u \in \Gamma, \forall g \in G .
$$

The inverse mapping $\widehat{f} \rightarrow f$ is defined as follows

$$
f(u)=\operatorname{tr}\{\widehat{f} \widehat{\Omega}(u)\} .
$$

Besides $\widehat{1}=I_{\mathcal{H}}$ and the traciality condition are equivalent to

$$
\operatorname{tr}\{\widehat{f} \widehat{g}\}=\int_{\Gamma} f(u) g(u) d u=\int_{\Gamma}(f \star g)(u) d u,
$$

with the Moyal product defined by

$$
(f \star g)(u)=\int_{\Gamma} d v \int_{\Gamma} d w \operatorname{tr}\{\widehat{\Omega}(u) \widehat{\Omega}(v) \widehat{\Omega}(w)\} f(v) g(w) .
$$

Note that the weak point of this quantization method is the lack of general theorems about existence and uniqueness of the Moyal quantizer.

\section{Moyal quantization on the cylinder via Weil-Brezin-Zak transform}

\subsection{The Weil-Brezin-Zak (WBZ) transform}

The Weil-Brezin-Zak (WBZ) transform is well known in solid state theory where it gives rise to the Bloch functions [27]. It is a unitary map $T: L^{2}(\mathbb{R}) \rightarrow L^{2}\left(S^{1} \times S^{1 *}\right)$ defined by

$$
(T \psi)(q, k)=\sum_{n=-\infty}^{\infty} e^{i n a k} \psi(q-n a),
$$

where $S^{1 *}=[0,2 \pi / a)$ is the dual space of $S^{1}=[0, a), \psi \in L^{2}(\mathbb{R}), q \in S^{1}$ and $k \in S^{1 *}$. Functions $T \psi(q, k)$ are periodic in $k$ and quasiperiodic in $q$

$$
(T \psi)\left(q+n a, k+m \frac{2 \pi}{a}\right)=e^{i n a k}(T \psi)(q, k), \quad n, m \in \mathbb{Z} .
$$

Fixing a value of $k$ the operator $T$ (hereafter denoted by $T^{(k)}$ ) is a projection onto $L^{2}\left(S^{1}\right)$. Thus, $\psi^{(k)}=T^{(k)} \psi$ is the decomposition of $\psi$ and we obtain the constant fiber direct integral decomposition [27],

$$
L^{2}(\mathbb{R}) \cong \int_{S^{1 *}}^{\oplus} d k L^{2}\left(S^{1}\right) .
$$

Let $A$ be an operator on $L^{2}(\mathbb{R})$. If there exists a function $A^{(\cdot)}: S^{1 *} \rightarrow \mathcal{L}\left(L^{2}\left(S^{1}\right)\right)$ such that

$$
(A \psi)^{(k)}=A^{(k)} \psi^{(k)}, \quad \forall \psi \in L^{2}(\mathbb{R})
$$


it is said that $A$ is "decomposable" and we can write

$$
A=\int_{S^{1 *}}^{\oplus} d k A^{(k)}
$$

Operators $A^{(k)}$ are called the "fibers" of $A$.

It is easy to prove that an operator $A$ in $L^{2}(\mathbb{R})$ is decomposable if and only if it is invariant under the translation $T_{a}$, i.e., $T_{a} \mathrm{~A} T_{a}^{-1}=A$.

The definition of decomposable operator works for bounded operators but it is possible to extend it to unbounded self-adjoint operators [27].

If $A^{(k)}$ is self-adjoint for each $k$, we have

$$
F(A)=\int_{S^{1 *}}^{\oplus} d k F\left(A^{(k)}\right)
$$

for any bounded function $F$ on $\mathbb{R}$.

Examples of decomposable operators are: the momentum operator $\widehat{P}$, the translation operator $T_{x}=\exp (i x \widehat{P} / h)$ and $E=\exp (i 2 \pi \widehat{Q} / a)$.

\subsection{Weyl correspondence via WBK transform}

The approach to quantization on the cylinder presented in this subsection is based on the fact that $L^{2}(\mathbb{R})$ is isomorphic to $L^{2}\left(S^{1} \times S^{1 *}\right)$.

From this point of view, decomposition of operators can be considered as the natural link between quantum theories on the circle and on the real line. That is because decomposable operators are operators on $L^{2}(\mathbb{R})$ which are invariant under the translation of length $a$, so they are really acting on $L^{2}\left(S^{1}\right)$. This is the case of operators $\widehat{f}$ associated with periodic functions in the position variable (we can of course consider $f$ as a function (or distribution) on the phase space $S^{1} \times \mathbb{R}$ ) [26].

The WBZ transform allows us to quantize on $S^{1} \times \mathbb{R}$ in a natural way as a particular case of quantization on $\mathbb{R} \times \mathbb{R}$. Functions $f(q, p)$ defined on the cylinder are periodic in the position variable $q \in[0, a)$. The Weyl quantization on $S^{1} \times \mathbb{R}$ is carried by the

fibers $\widehat{f}^{(k)}$ of the operator $\widehat{f}$, defined by (2.3). Since in this case $f(q, p)$ is periodic in $q$, operator $\widehat{f}$ yields

$$
\widehat{f}=\frac{1}{2 \pi \hbar} \int_{-\infty}^{\infty} d x \sum_{n=-\infty}^{\infty} \tilde{f}_{n}(x) e^{i(2 \pi n \widehat{Q} / a+x \widehat{P} / \hbar)},
$$

where

$$
\widetilde{f}_{n}(x):=\int_{0}^{a} d q f(q, p) \exp (-i q n / \hbar) .
$$

This operator can be seen as an integral operator

$$
(\widehat{f} \psi)(x)=\int_{-\infty}^{\infty} K_{f}(x, y) \psi(y) d y, \quad \psi \in \mathcal{S}(\mathbb{R})
$$


with integral kernel

$$
\begin{aligned}
K_{f}(x, y) & =\frac{1}{2 \pi \hbar} \sum_{n=-\infty}^{\infty} \widetilde{f}_{n}(y-x) e^{i \pi n(x+y) / a} \\
& =\frac{1}{2 \pi \hbar} \int_{-\infty}^{\infty} f\left(\frac{1}{2}(x+y), p\right) e^{i(x-y) p / \hbar} d p
\end{aligned}
$$

From the second equation (questions about domains and convergence can be solved appealing to suitable conditions on $f$ [26]) we arrive to the following intermediate expression

$$
\begin{array}{r}
(\widehat{f} \psi)^{(k)}(q)=\frac{1}{2 \pi \hbar} \int_{-\infty}^{\infty} d p \int_{-\infty}^{\infty} d x f\left(\frac{1}{2}(q+x), p\right) \\
\times e^{i(q-x) p / \hbar} e^{i a k[x / a]} \psi^{(k)}\left((x)_{a}\right),
\end{array}
$$

where $x=(x)_{a}+a[x / a]$ and $[y]$ the integer part of the real number $y$. Finally, we obtain the operator in $S^{1} \times \mathbb{R}$ given by

$$
\widehat{f}^{(k)}=\frac{1}{2 \pi \hbar} \int_{-\infty}^{\infty} d x \sum_{n=-\infty}^{\infty} \widetilde{f}_{n}(x) e^{i \pi n x / a} E^{n} \exp \left(i x P^{(k)} / \hbar\right),
$$

which can be seen as an integral operator on $L^{2}\left(S^{1}\right)$ (under suitable conditions on the function $f$ on $S^{1} \times \mathbb{R}$ )

$$
\left(\widehat{f}^{(k)} \varphi\right)(q)=\int_{0}^{a} K_{f}^{(k)}\left(q, q^{\prime}\right) \varphi\left(q^{\prime}\right) d q^{\prime}
$$

with integral kernel

$$
\begin{aligned}
& K_{f}^{(k)}\left(q, q^{\prime}\right)=\left(T^{(k)} K_{f}\left(\cdot, q^{\prime}\right)\right)(q) \\
& \quad=\frac{1}{2 \pi \hbar} \sum_{n=-\infty}^{\infty} e^{i n a k} \int_{-\infty}^{\infty} d p f\left(\frac{1}{2}\left(q+q^{\prime}-n a\right), p\right) e^{i\left(q-q^{\prime}-n a\right) p / \hbar}
\end{aligned}
$$

The operator (3.6) has following properties:

(i) $\widehat{f}^{(k) \dagger}=\widehat{f}^{*}(k)$,

(ii) if $f$ is a function only of $p$, then $\widehat{f}^{(k)}=f\left(P^{(k)}\right)$ as defined by the spectral theorem,

(iii) if $g$ is a function only of $q$, we know that $g(q)=\sum_{n=-\infty}^{\infty} \widetilde{g}_{n} e^{i 2 \pi n q / a}$. Then $\widehat{g}^{(k)}=\sum_{n=-\infty}^{\infty} \widetilde{g}_{n} E^{n}$.

An interesting fact is that the operators in $L^{2}\left(S^{1}\right)$ appearing in (3.6)

$$
U^{(k)}(t, x, n):=e^{i \pi(2 t+n x) / a} E^{n} e^{i x P^{(k)} / \hbar}
$$

verify the following statements:

(i) It determines a unitary irreducible representation of $\left\{\left(t, x, \frac{2 \pi}{a} \hbar n\right) \mid t, x \in \mathbb{R}, n \in\right.$ $\mathbb{Z}\}$, subgroup of the Heisenberg group.

(ii) The operators $U^{(k)}(x, n):=U^{(k)}(0, x, n)$ carry a unitary irreducible realization (i.e. up to a factor representation) of the subgroup $\left\{\left(x, \frac{2 \pi}{a} \hbar n\right) \mid x \in S^{1}, n \in \mathbb{Z}\right\}$ of the abelian group $S^{1} \times \mathbb{R}$. 
The proof can be done by considering the Schrödinger representation of the Heisenberg group given by the operators

$$
U_{\lambda}(t, y, p):=e^{i \lambda t I} \exp \left(\frac{i}{\hbar}(p \widehat{Q}-y \widehat{P})\right),
$$

where $\lambda$ is a real parameter labelling the representation, and that the operators $U_{\lambda}(t, y, p)$ are only decomposable when $p=2 \pi \hbar n / a$ with $n \in \mathbb{Z}$. The operators $U^{(k)}(t, x, n)$ are obtained decomposing the operators $U_{2 \pi / a}(t,-x, 2 \pi \hbar n / a)$.

\subsection{Coherent states on the circle and Weyl quantization}

Coherent states (CS) in $L^{2}\left(S^{1}\right)$ can be constructed [34, 35] by decomposition of the standard Weyl-Heisenberg $(\mathrm{WH}) \mathrm{CS}$ in $L^{2}(\mathbb{R})[13,36]$

$$
\eta_{q, p}(x)=\exp \left(\frac{i}{\hbar} p\left(x-\frac{q}{2}\right)\right) \eta_{0}(x-q), \quad x, q, p \in \mathbb{R}, \eta_{0} \in L^{2}(\mathbb{R}),
$$

by the WBZ transform (3.1)

$$
\eta_{q, p}^{(k)} \equiv|q, p ; k\rangle:=T^{(k)} \eta_{q, p}, \quad(q, p) \in S^{1} \times \mathbb{R} .
$$

They verify the resolution of unity

$$
\frac{1}{2 \pi \hbar} \int_{0}^{a} d q \int_{-\infty}^{\infty} d p|q, p ; k\rangle\langle q, p ; k|=\widehat{I} .
$$

Choosing the fiducial state $\eta_{0} \in L^{2}(\mathbb{R})$ to be a normalized Gaussian,

$$
\eta_{0}(x)=\left(\frac{\omega}{\pi \hbar}\right)^{1 / 4} \exp \left(-\frac{\omega}{2 \hbar} x^{2}\right),
$$

the CS's on the circle take the form

$$
\begin{aligned}
\eta_{q, p}^{(k)}\left(q^{\prime}\right)=\left(\frac{\omega}{\pi \hbar}\right)^{1 / 4} & \exp \left(\frac{i}{2 \omega \hbar} p z^{*}\right) \exp \left(-\frac{1}{2 \omega \hbar}\left(z^{*}-\omega q^{\prime}\right)^{2}\right) \\
\times & \theta\left(i \frac{a}{2 \hbar}\left(z^{*}-\omega q^{\prime}-i k \hbar\right) ; \rho_{1}\right),
\end{aligned}
$$

with $z^{*}=\omega q+i p, \rho_{1}=\exp \left(-a^{2} \omega /(2 \hbar)\right)$ and $\theta(z ; \rho)=\sum_{n=-\infty}^{\infty} \rho^{n^{2}} e^{2 i n z},|\rho|<1$. By $\theta(z ; \rho)$ we denote the Theta function (sometimes written as $\theta_{3}$ ) $[38,39]$.

Since these CS have been constructed by decomposition of the standard WH CS in $L^{2}(\mathbb{R})$, we can compare both kinds of CS. The physical properties of the CS on the circle depend on some dimensionless parameter, related to the spread of the initial WH-CS. If it is smaller than the length $a$ of the circle, the CS on the circle are very similar to the WH-CS. But when such spread is comparable or bigger than $a$, the CS on the circle are rather like plane waves. A detailed study of the properties of these CS can be found in [37]. For instance, the expectation values of the position and momentum are obtained as well as the Heisenberg uncertainty relation. In [40] a particular case of these CS were introduced by Kowalski et al to study a quantum particle on the circle. Recently in [41] Kowalski and Rembieliński have introduced new uncertainty relations for the position and momentum on the circle using the above mentioned CS. 
The Weyl correspondence on the cylinder (3.6) also presents a correct semiclassical behaviour $[34,35]$ For this purpose the CS on the circle $|q, p ; k\rangle$ are used for computing the expectation values of the operators in these states. Also the limit of these expectation values when $\hbar$ goes to 0 is taken. We display the final results (see [35] for details). Most of the computations have been made using the functional equation of $\theta[38,39]$. So, one previously gets

$$
\begin{aligned}
& \lim _{\hbar \rightarrow 0}\langle q, p ; k \mid q, p ; k\rangle=1, \\
& \lim _{\hbar \rightarrow 0} \frac{1}{2 \pi \hbar}\left|\left\langle q^{\prime}, p^{\prime} ; k \mid q, p ; k\right\rangle\right|^{2}=\delta\left(q^{\prime}-q\right) \delta\left(p^{\prime}-p\right) .
\end{aligned}
$$

The second expression shows that the CS $|q, p ; k\rangle$ concentrates at the phase space point $(q, p)$ in the semiclassical limit. The expectation values of the operators $E$ and $P^{(k)}$ are, respectively,

$$
\begin{aligned}
& \lim _{\hbar \rightarrow 0}\langle q, p ; k|E| q, p ; k\rangle=e^{i 2 \pi q / a}, \\
& \lim _{\hbar \rightarrow 0}\left\langle q, p ; k\left|P^{(k)}\right| q, p ; k\right\rangle=p .
\end{aligned}
$$

After some cumbersome but straightforward calculations, it is proved that, if $\widehat{f}^{(k)}$ is the operator associated by the Weyl application (3.6) to the function $f(q, p)$ on $S^{1} \times \mathbb{R}$, then

$$
\begin{aligned}
& \lim _{\hbar \rightarrow 0}\left\langle q, p ; k\left|\widehat{f}^{(k)}\right| q, p ; k\right\rangle=f(q, p), \\
& \lim _{\hbar \rightarrow 0} \frac{1}{i \hbar}\left\langle q, p ; k\left|\left[\widehat{f}^{(k)}, \widehat{g}^{(k)}\right]\right| q, p ; k\right\rangle=\{f, g\}(q, p),
\end{aligned}
$$

where $\{f, g\}$ is the Poisson bracket of the functions $f, g$ on $S^{1} \times \mathbb{R}$.

It is worthy noting that these CS have not been constructed by Perelomov's method [13], as an orbit under a Lie group representation. They constitute a non-trivial example of the "reproducing triplets" introduced in [42]. In reality, there is not a group generating these CS. This is related to the fact that the Schrödinger representation is not decomposable.

The action of the operators $U^{(k)}(x, n)$ over the CS $|q, p ; k\rangle$ is

$$
U^{(k)}(x, n)|q, p ; k\rangle=e^{i \pi n q / a} e^{i p x /(2 \hbar)}\left|q-x, p+\frac{2 \pi}{a} \hbar n ; k\right\rangle .
$$

All that has strong consequences on the physical interpretation of the parameters $q$ and $p$. In the case of the WH-CS the parameters of the "classical" phase space $\mathbb{R}^{2}$ are in correspondence with the expectation values of the operators $\widehat{Q}$ and $\widehat{P}$. The Schrödinger representation, generating these CS, acts by translations over the expectation values, which constitute a true "quantum" phase space coinciding in this case with the classical phase space. However, the CS on the cylinder, $|q, p ; k\rangle$, are very different, since the luck of a group generating these CS does not allows us to give a clear physical interpretation for $q$ and $p$. In fact, it is known that the cylinder cannot be seen as a quantum phase space with translation operators like those of $\mathbb{R}^{2}$ because the discrete 
nature of the spectrum of the momentum operators in $L^{2}\left(S^{1}\right)$. Only the subgroup $\left\{(x, 2 \pi \hbar n / a) \mid x \in S^{1}, n \in \mathbb{Z}\right\}$ of $S^{1} \times \mathbb{R}$ can be used as quantum phase space, being $U^{(k)}(x, n)$ the corresponding translation operators. This result is in agreement with the conclusions obtained by other authors, for instance [21, 24]. Obviously, the parameters $q$ and $p$ do not coincide in general with the expectation values of the position and momenta operators for the CS $|q, p ; k\rangle$.

\subsection{Weyl symbols on the cylinder}

The Weyl application (2.3) on $\mathbb{R}^{2}$ is one-to-one, so it can be inverted. The function (or symbol)

$$
f=\operatorname{Tr}\{\widehat{f} \widehat{\Omega}\}
$$

is associated with each operator $\widehat{f}$. These symbols are useful to develop the phase space formalism of quantum theory $[5,6,7]$. However, the Weyl correspondence on the cylinder (3.6) is not one-to-one, hence symbols on $S^{1} \times \mathbb{R}$ cannot be defined in this way.

Let $\alpha_{n}$ be the Fourier coefficients of the function $e^{-i k q} \phi(q)$, where $\phi \in L^{2}\left(S^{1}\right)$ is an arbitrary function and $k \in[0,2 \pi / a)$ fixed,

$$
\begin{aligned}
\phi(q) & =\sum_{n=-\infty}^{\infty} \alpha_{n} e^{i(2 \pi n / a+k) q}, \\
\alpha_{n} & =\frac{1}{a} \int_{S^{1}}^{1} d q \phi(q) e^{-i(2 \pi n / a+k) q} .
\end{aligned}
$$

There exists at least one function $\psi \in L^{2}(\mathbb{R})$ such that $\phi=\psi^{(k)}$, i.e., $\psi$ will be some function of $L^{2}(\mathbb{R})$ verifying

$$
\alpha_{n}=\frac{1}{a} \widetilde{\psi}\left(\left(\frac{2 \pi}{a} n+k\right) \hbar\right) .
$$

On the other hand, we can write

$$
\begin{aligned}
\frac{1}{a} \widetilde{\psi}((2 \pi n / a & +k) \hbar)=\frac{1}{a} \int_{R} d x \psi(x) e^{-i(2 \pi n / a+k) x} \\
= & \frac{1}{a} \sum_{a \in \mathbb{Z}} \int_{S^{1}} d q \psi(q-a) e^{i a k} e^{-i(2 \pi n / a+k) q} \\
= & \frac{1}{a} \int_{S^{1}} d q\left(\sum_{a \in \mathbb{Z}} e^{i a k} \psi(q-a)\right) e^{-i(2 \pi n / a+k) q} \\
= & \frac{1}{a} \int_{S^{1}} d q \psi(q) e^{-i(2 \pi n / a+k) q} .
\end{aligned}
$$

Hence, we have derived the expression for the Fourier coefficients of the function $e^{-i k q} \psi^{(k)}(q)$. Since $\psi$ verifies relations (3.14), then $\psi^{(k)}=\phi$ due to the uniqueness of the Fourier coefficients. There are many functions $\psi$ that verify (3.14) for some fixed $\alpha_{2 \pi n / a}$. In other words, given $\phi$, the function $\psi$, such that $\psi^{(k)}=\phi$, is not uniquely determined. The factor $e^{-i k q}$ has been introduced because the functions $\psi^{(k)}(q)$, obtained by using the expression (3.1), are quasiperiodic in $q \in \mathbb{R}$. Multiplying (3.1) by $e^{-i k q}$ we obtain functions strictly periodic. The usual Fourier analysis can be applied to them. 
Quantum Mechanics on the cylinder

The second formula of (3.13) shows that two functions $\psi_{1}, \psi_{2} \in L^{2}(\mathbb{R})$ have the same image under $T^{(k)}$ if

$$
\widetilde{\psi}_{1}\left(\left(\frac{2 \pi}{a} n+k\right) \hbar\right)=\widetilde{\psi}_{2}\left(\left(\frac{2 \pi}{a} n+k\right) \hbar\right), \quad \forall n \in \mathbb{Z} .
$$

The integral kernel $K_{f}^{(k)}\left(q, q^{\prime}\right)$ of a Weyl operator $\widehat{f}^{(k)}$ in $L^{2}\left(S^{1}\right)$ is the image, as function of $q$, under $T^{(k)}$ of the integral kernel $K_{f}$ of the corresponding Weyl operator $\widehat{f}$ in $L^{2}(\mathbb{R})$. Then, two functions $f$ and $g$ on $S^{1} \times \mathbb{R}$ will be associated with the same operator on $L^{2}\left(S^{1}\right)$ if

$$
\widetilde{K}_{f}^{1)}\left(\left(\frac{2 \pi}{a} n+k\right) \hbar, q^{\prime}\right)=\widetilde{K}_{g}^{1)}\left(\left(\frac{2 \pi}{a} n+k\right) \hbar, q^{\prime}\right), \quad \forall n \in \mathbb{Z}, q^{\prime} \in S^{1},
$$

where $\widetilde{K}_{f}^{1)}$ denotes the partial Fourier transform of $K_{f}$ with respect to its first variable. After a cumbersome computation we get

$$
\begin{aligned}
\widetilde{K}_{f}^{1)}\left(\left(\frac{2 \pi}{a} n+k\right) \hbar, y\right)= & \frac{1}{a} e^{i(2 \pi n / a-k) y} \sum_{m=-\infty}^{\infty} e^{-i 2 \pi m y / a} \\
& \times \int_{0}^{a} d q f\left(q,\left(\frac{\pi}{a} m+k\right) \hbar\right) e^{i 2 \pi(m-2 n) q / a} .
\end{aligned}
$$

Hence,

$$
\widehat{f}^{(k)}=\widehat{g}^{(k)} \Longleftrightarrow f\left(q,\left(\frac{\pi}{a} n+k\right) \hbar\right)=g\left(q,\left(\frac{\pi}{a} n+k\right) \hbar\right), \quad \forall n \in \mathbb{Z}, q \in S^{1} .
$$

Thus, it is sufficient that $f(q, p)$ and $g(q, p)$ coincide on a discrete set of values of $p$, depending on $\hbar$ to be the symbols of an operator on the cylinder. This observation is basic for a correct definition of the symbols associated with operators on the cylinder.

It is possible to define symbols of the operators $\widehat{f}^{(k)}$ for a quantum phase space (the cylinder) different from the classical one as it is showed in the following [35].

The integral kernel (3.8) of $\widehat{f}^{(k)}$ can be rewritten as

$$
K_{f}^{(k)}\left(q, q^{\prime}\right)=e^{i k\left(q-q^{\prime}\right)} \sum_{n=-\infty}^{\infty} e^{i \pi\left(q-q^{\prime}\right) n / a} F\left(q+q^{\prime}, n\right),
$$

where

$$
F(x, n):=\frac{1}{2 a}\left\{f\left(\frac{x}{2},\left(\frac{\pi}{a} n+k\right) \hbar\right)+(-1)^{n} f\left(\frac{x+a}{2},\left(\frac{\pi}{a} n+k\right) \hbar\right)\right\} .
$$

From relation (3.16) we deduce that there is a one-to-one correspondence between the operators $\widehat{f}^{(k)}$ with integral kernel $K_{f}^{(k)}\left(q, q^{\prime}\right)$ and the functions $F(x, n)$. Hence, $F(x, n)$ is taken as the Weyl symbol of $\widehat{f}^{(k)}$. To see this relationship more clearly we can rewrite (3.16), using the new variables $x:=q+q^{\prime}, 2 y:=q-q^{\prime}$, as

$$
K_{f}^{(k)}\left(\frac{x}{2}+y, \frac{x}{2}-y\right)=e^{i 2 k y} \sum_{n=-\infty}^{\infty} e^{i 2 \pi y n / a} F(x, n) .
$$


This equation can be inverted

$$
F(x, n)=\frac{1}{a} \int_{0}^{a} d y e^{-i 2(\pi n / a+k) y} K_{f}^{(k)}\left(\frac{x}{2}+y, \frac{x}{2}-y\right) .
$$

Note that the symbol $F(x, n)$ only depends on the value of the function $f(q, p)$ for a discrete set of values of $p$. The (non bijective) correspondence between $F(x, n)$ and $f(q, p)$ given by (3.17) can be seen as an alternative expression of the Weyl quantization rule on the cylinder.

The property

$$
F(x+a, n)=(-1)^{n} F(x, n),
$$

allows us to consider the set $\left\{(q, n) \mid q \in S^{1}, n \in \mathbb{Z}\right\}$ as the quantum phase space where the symbols are defined. It is worthy noticing that the symbols $F(x, n)$ are periodic in $x$ with period $a$ when $n$ is odd and period $2 a$ when $n$ is even, but the classical functions $f(q, n)$ are periodic in $q$ with period $a$.

\subsection{The Moyal quantizer}

As we mention before the basic problem of Moyal's formalism is the existence of the Moyal quantizer $\widehat{\Omega}$. It is usually solved using group theory, but this method does not work in the case of the cylinder because we have not a symmetry group. However, the existence of the Weyl correspondence allows us to construct $\widehat{\Omega}$ and reformulate the Weyl quantization as Moyal quantization [35].

According to the definition of the Moyal quantization (expression (2.12)) in the case of the cylinder we write

$$
\widehat{f}^{(k)}=\int_{-\infty}^{\infty} d p \int_{0}^{a} d q f(q, p) \widehat{\Omega}^{(k)}(q, p),
$$

with $\widehat{f}^{(k)}$ being the operator associated with the classical function $f(q, p)$ by the Weyl correspondence $(2.5)$ and $\widehat{\Omega}^{(k)}$ the cylinder Moyal quantizer. But the Weyl correspondence does not verify the bijectivity requirement, so we are compelled to use the symbols $F(q, n)$ instead the classical functions $f(q, p)$. Hence, we have

$$
\widehat{f}^{(k)}=\sum_{n=-\infty}^{\infty} \int_{0}^{a} d q F(q, n) \widehat{\Omega}^{(k)}(q, n) .
$$

So, $\widehat{\Omega}^{(k)}$ has to be defined on the quantum phase space $S^{1} \times \mathbb{Z}$ as it happens with the symbols $F$.

Although the operators $\widehat{f}^{(k)}$ were constructed by decomposition of the Weyl operators in $L^{2}(\mathbb{R})$, the corresponding Moyal quantizer cannot be obtained in the same way since the Grossmann-Royer operators (2.6), that determine the Moyal quantizer in $\mathbb{R}^{2}$, are not decomposable.

Starting from expression (3.5) and after some calculations we obtain

$$
\left(\widehat{\Omega}^{(k)}(x, n) \varphi\right)(q)=e^{i k(2 q-x)} e^{i \pi(2 q-x) n / a} \varphi_{c}(x-q), \quad \varphi \in L^{2}\left(S^{1}\right),
$$


where $\varphi_{c}(x)=e^{i a k[x / a]} \varphi\left(x-a\left[\frac{x}{a}\right]\right)$.

We must check if the operators (3.21) fulfill the conditions to be a Moyal quantizer displayed in section (2.2). The demonstration of that $\widehat{\Omega}^{(k)}$ is bijective and self-adjoint is easy and we let it for the reader. The proof of that $\operatorname{tr} \widehat{\Omega}(u)=1$ and traciality (2.13) requires to use an appropriate orthonormal basis of $L^{2}\left(S^{1}\right),\{|n ; k\rangle \mid n \in \mathbb{Z}, k \in$ $\left(S^{1}\right)^{\prime}$ fixed $\}$, given by

$$
\langle q \mid n ; k\rangle=\frac{1}{\sqrt{a}} \exp \left[i\left(\frac{2 \pi}{a} n+k\right) q\right] .
$$

After a straightforward computation we get

$$
\operatorname{tr} \widehat{\Omega}^{(k)}(x, n)=\frac{1}{2}\left(1+(-1)^{n}\right),
$$

and

$$
\operatorname{tr}\left\{\widehat{\Omega}^{(k)}(x, n) \widehat{\Omega}^{(k)}(y, m)\right\}=a \delta_{n, m} \delta(x-y)
$$

Note that $\widehat{\Omega}^{(k)}$ is not a proper Moyal quantizer since the property of trace unit is not accomplished.

It is worthy to mention that since $\widehat{f}^{(k)}$ is an integral operator with kernel $K_{f}^{(k)}$ and supposing that it is of trace class, we can prove that

$$
\operatorname{tr} \widehat{f}^{(k)}=\int_{0}^{a} d q K_{f}^{(k)}(q, q)=\sum_{n=-\infty}^{\infty} \int_{0}^{a} d q F(2 q, n),
$$

and

$$
\operatorname{tr} \widehat{f}^{(k)}=\sum_{n=-\infty}^{\infty} \int_{0}^{a} d q F(q, n) \operatorname{tr} \widehat{\Omega}^{(k)}(q, n)=\sum_{n=-\infty}^{\infty} \int_{0}^{a} d q F(q, 2 n) .
$$

On the other hand, the symbol associated with the function $f \equiv 1$, i.e. to the unit operator in $L^{2}\left(S^{1}\right)$, is

$$
F_{1}(n)=\frac{1}{2 a}\left(1+(-1)^{n}\right) .
$$

This result is due to the fact that $\operatorname{tr} \widehat{\Omega}^{(k)} \neq 1$. The classical function $f \equiv 1$ is associated with the identity operator although its corresponding quantum symbol is not 1 . This last result is intrinsic of this formalism.

Despite $\operatorname{tr} \widehat{\Omega}^{(k)} \neq 1$ a quantum formalism can be developed in the cylinder as phase space. The more relevant formulas for it are (2.14)-(2.16) and only those that dependent on $\operatorname{tr} \widehat{\Omega}^{(k)}$ have to be modified. Thus, formula (2.14) as well as the first equality of (2.15) are valid, since they are only based on the tracial property (3.23). We have in these cases

$$
\begin{aligned}
& F(q, n)=\frac{1}{a} \operatorname{tr}\left\{\widehat{f}^{(k)} \widehat{\Omega}^{(k)}(q, n)\right\} \\
& \operatorname{tr}\left\{\widehat{f}^{(k)} \widehat{g}^{(k)}\right\}=a \sum_{n=-\infty}^{\infty} \int_{0}^{a} d q F(q, n) G(q, n),
\end{aligned}
$$


with $F$ and $G$ the symbols of $\widehat{f}^{(k)}$ and $\widehat{g}^{(k)}$, respectively. Likewise, the expression of the twisted product of two symbols (2.16) is directly applicable in this case. With the help of formula (3.27) we obtain

$$
\begin{aligned}
(F \star G)(x, n)= & \frac{1}{a} \operatorname{tr}\left\{\widehat{f}^{(k)} \widehat{g}^{(k)} \widehat{\Omega}^{(k)}(x, n)\right\} \\
= & \frac{1}{a} \sum_{\substack{m=-\infty \\
l=-\infty \\
l}}^{\infty} \sum_{0}^{\infty} d y \int_{0}^{a} d z F(y, m) G(z, l) \\
& \quad \operatorname{tr}\left\{\widehat{\Omega}^{(k)}(x, n) \widehat{\Omega}^{(k)}(y, m) \widehat{\Omega}^{(k)}(z, l)\right\},
\end{aligned}
$$

where

$$
\begin{aligned}
& \operatorname{tr}\left\{\widehat{\Omega}^{(k)}(x, n) \widehat{\Omega}^{(k)}(y, m) \widehat{\Omega}^{(k)}(z, l)\right\} \\
& \quad=\frac{1}{2}\left(1+(-1)^{n-m+l}\right) e^{i \pi(m-l) x / a} e^{i \pi(l-n) y / a} e^{i \pi(n-m) z / a} .
\end{aligned}
$$

However, the second equality of (2.15) is not valid and it has to be substituted by

$$
\begin{aligned}
\operatorname{tr}\left\{\widehat{f}^{(k)} \widehat{g}^{(k)}\right\} & =\sum_{n=-\infty}^{\infty} \int_{0}^{a} d q(F \star G)(q, n) \operatorname{tr} \widehat{\Omega}^{(k)}(q, n) \\
& =\sum_{n=-\infty}^{\infty} \int_{0}^{a} d q(F \star G)(q, 2 n)
\end{aligned}
$$

where we have used (3.22). Expression (3.31) as well as (3.25) are related to the fact that the symbol $F_{1}(n)$, given by (3.26), plays the role of unit function in the quantum formalism on phase space. Note that $\operatorname{tr} \widehat{\Omega}^{(k)}(q, n)=a F_{1}(n)$.

\section{Moyal quantization on the cylinder via unitary operators}

The quantization procedure, that we present now, has been developed in ref. [24]. It is based on results of $[17,18]$ and we will call it the PPTT formalism. Let $(\theta, p)$ be the coordinates on the cylinder: $\theta \in[-\pi, \pi)$ and $p \in(-\infty, \infty)$ (the angular momentum conjugate to $\theta$ ).

Let us consider the family of unitary operators

$$
\widehat{U}(\tau, m)=\exp \left\{i\left(\frac{\tau}{\hbar} \widehat{p}+m \widehat{\theta}\right)\right\}, \quad m \in \mathbb{Z},-\pi \leq \tau<\pi .
$$

The following property holds

$$
\operatorname{Tr}\left\{\widehat{U}^{+}(\tau, m) \widehat{U}\left(\tau^{\prime}, m^{\prime}\right)\right\}=2 \pi \delta_{m, m^{\prime}} \delta^{(S)}\left(\tau-\tau^{\prime}\right)
$$

where $\delta^{(S)}(\theta)$ denotes the Dirac delta on the circle $S^{1}$.

Let $f=f(\theta, p)$ be a function on the cylinder and $\widehat{f}$ some linear operator representing $f$ in the quantum case. Expanding $\widehat{f}$ with respect to the basis (4.1) one gets

$$
\widehat{f}=\frac{1}{(2 \pi)^{2}} \sum_{m=-\infty}^{\infty} \int_{-\pi}^{\pi} \tilde{f}(\tau, m) \widehat{U}(\tau, m) d \tau,
$$

where $\tilde{f}(\tau, m)$ is a function to be determined. From (4.3) and (4.2) one obtains that

$$
\tilde{f}(\tau, m)=2 \pi \operatorname{Tr}\left\{\widehat{U}^{+}(\tau, m) \widehat{f}\right\} .
$$


A consequence of expression (4.4) is that the operator $\widehat{f}$ determines a function $\tilde{f}(\tau, m)$ only for $\tau \in[-\pi, \pi)$. It means that if it is assumed (by analogy with $\mathbb{R}^{2}$ ) that $\tilde{f}(\tau, m)$ is the Fourier transform of $f(\theta, p)$, one is not able to extract all the information about this transform from (4.3) but only its values in the interval $[-\pi, \pi)$.

Considering the following distribution on the cylinder $S^{1} \times \mathbb{R}$

$$
F(\theta, p):=\sum_{n=-\infty}^{\infty} f(\theta, n \hbar) \delta(p-n \hbar),
$$

its Fourier transform, $\widetilde{F}(p, \theta)$, is defined by

$$
\begin{aligned}
\widetilde{F}(\theta, p)(\tau, m) & =\int_{-\infty}^{\infty} d p \int_{-\pi}^{\pi} d \theta F(p, \theta) \exp \left\{-i\left(\frac{\tau}{\hbar} p+m \theta\right)\right\} \\
& =\sum_{n=-\infty}^{\infty} \int_{-\pi}^{\pi} d \theta f(\theta, n \hbar) \exp \{-i(\tau n+m \theta)\} .
\end{aligned}
$$

The quantization rule (4.3) applied together with (4.5) and (4.6) constitutes a oneto-one correspondence between functions on $S^{1} \times \hbar \mathbb{Z} \subset S^{1} \times \mathbb{R}$ and operators on the Hilbert space $L^{2}\left(S^{1}\right)$.

Substituting the second equality of (4.6) in (4.3) it is obtained that

$$
\widehat{f}=\sum_{n=-\infty}^{\infty} \int_{-\pi}^{\pi} \frac{d \theta}{2 \pi} f(\theta, n \hbar) \widehat{\Omega}(\theta, n),
$$

where

$$
\widehat{\Omega}(\theta, n):=\frac{1}{2 \pi} \sum_{m=-\infty}^{\infty} \int_{-\pi}^{\pi} d \tau \exp \{-i(\tau n+m \theta) \widehat{U}(\tau, m),
$$

or, in an alternative form

$$
\widehat{\Omega}(\theta, n)=\int_{-\pi}^{\pi} \exp (i \tau n)\left|\left[\theta+\frac{\tau}{2}\right]\right\rangle\left\langle\left[\theta-\frac{\tau}{2}\right]\right| d \tau .
$$

The operator $\widehat{\Omega}(n, \theta)$ is a Moyal quantizer since it verifies all the required conditions. Notice that it is defined not on the whole cylinder $S^{1} \times \mathbb{R}$ but only on $S^{1} \times \mathbb{Z}$.

Obviously, from (4.7) one recovers $f$

$$
f(\theta, n \hbar)=\operatorname{Tr}\{\widehat{\Omega} \widehat{f}\} .
$$

The $*$-product of functions on the cylinder associated with this quantizer is given by

$$
\begin{aligned}
& \left(f_{1} * f_{2}\right)(\theta, n \hbar)=\operatorname{Tr}\left\{\widehat{\Omega}(\theta, n) \widehat{f_{1}} \widehat{f}_{2}\right\}=\frac{1}{4 \pi^{2}} \sum_{n^{\prime}, n^{\prime \prime}=-\infty}^{\infty} \int_{-\pi}^{\pi} d \theta^{\prime} \int_{-\pi}^{\pi} d \theta^{\prime \prime} \\
& \quad \times\left\{f_{1}\left(\theta^{\prime}, n^{\prime} \hbar\right) \operatorname{Tr}\left\{\widehat{\Omega}(\theta, n) \widehat{\Omega}\left(\theta^{\prime}, n^{\prime}\right) \widehat{\Omega}\left(\theta^{\prime \prime}, n^{\prime \prime}\right)\right\} f_{1}\left(\theta^{\prime \prime}, n^{\prime \prime} \hbar\right)\right\}
\end{aligned}
$$


where $f_{1}(\theta, n \hbar)$ and $f_{2}(\theta, n \hbar)$ are arbitrary functions on $S^{1} \times \mathbb{Z}$ and $\widehat{f_{1}}, \widehat{f_{2}}$ their corresponding operators. The explicit expression of the trikernel is

$$
\begin{aligned}
\operatorname{Tr}\left\{\widehat{\Omega}(\theta, n) \widehat{\Omega}\left(\theta^{\prime}, n^{\prime}\right) \widehat{\Omega}\left(\theta^{\prime \prime}, n^{\prime \prime}\right)\right\}=\exp \left\{2 i\left[\left(n^{\prime \prime}-n\right)\left(\theta^{\prime}-\theta\right)-\left(n^{\prime}-n\right)\left(\theta^{\prime \prime}-\theta\right)\right]\right\} \\
\times\left\{1+\operatorname{sgn}\left(\cos \left(\theta^{\prime \prime}-\theta\right)\right) \operatorname{sgn}\left(\cos \left(\theta^{\prime}-\theta\right)\right)+\operatorname{sgn}\left(\cos \left(\theta^{\prime}-\theta\right)\right) \operatorname{sgn}\left(\cos \left(\theta^{\prime \prime}-\theta^{\prime}\right)\right)\right. \\
\left.\quad+\operatorname{sgn}\left(\cos \left(\theta^{\prime \prime}-\theta^{\prime}\right)\right) \operatorname{sgn}\left(\cos \left(\theta^{\prime \prime}-\theta\right)\right)\right\},
\end{aligned}
$$

where $\operatorname{sgn}(x)$ is equal to 1,0 or -1 if $x>0, x=0$ or $x<0$ respectively. Then, it is obtained the following expression for $f_{1} * f_{2}$

$$
\left(f_{1} * f_{2}\right)(\theta, n \hbar)=\left.\left\{f_{1}(\theta, p) \exp \left(\frac{i \hbar}{2} \stackrel{\leftrightarrow}{\mathcal{P}}\right) f_{2}(\theta, p)\right\}\right|_{p=n \hbar}
$$

with

$$
\overleftrightarrow{\mathcal{P}}=\frac{\overleftarrow{\partial}}{\partial \theta} \frac{\vec{\partial}}{\partial p}-\frac{\grave{\partial}}{\partial p} \frac{\vec{\partial}}{\partial \theta}
$$

The same result was obtained by Berezin [8].

\section{Conclusions and remarks}

As we have mentioned before there exist other quantization procedures to be applied to the cylinder as phase space. We shall prove that the methods proposed in ref. [21],[24] and our attempt are equivalent.

\subsection{Moyal quantization by WBZ versus PPTT formalism}

The crucial role in the quantization procedure proposed in [24] is played by a Moyal quantizer. Hence, to prove the equivalence of the quantum formalism introduced in ref. [24] and ours it is enough to demonstrate that formulas (3.20) and (4.7) are equivalent.

The action of the Moyal quantizer (4.9) on an arbitrary function $\varphi(\alpha)$ is

$$
(\widehat{\Omega}(\theta, n) \varphi)(\alpha)=\exp (2 i n(\alpha-\theta)) \varphi(2 \theta-\alpha) .
$$

Substituting $2 \theta$ by $\chi$ (now $\chi \in[-2 \pi, 2 \pi)$ ) we see that (5.1) equals

$$
\exp (i n(2 \alpha-\chi)) \varphi(\chi-\alpha) \text {. }
$$

This result (5.2) can be also obtained by the quantizer $\widehat{\Omega}^{(0)}(n, x)$ given by (3.21) with $k=0$ and $a=2 \pi$.

Now from expressions (4.7) and (5.2) we obtain

$$
\widehat{f}=\sum_{n=-\infty}^{\infty} \int_{-2 \pi}^{2 \pi} \frac{d x}{4 \pi} \widehat{\Omega}^{(0)}(x, n) f\left(\frac{x}{2}, n \hbar\right) .
$$


Splitting the above integral in a sum of three terms

$$
\begin{aligned}
\widehat{f}=\sum_{n=-\infty}^{\infty} \int_{-\pi}^{\pi} \frac{d x}{4 \pi} \widehat{\Omega}^{(0)}(x, n) f\left(\frac{x}{2}, n \hbar\right) \\
+\sum_{n=-\infty}^{\infty} \int_{-2 \pi}^{-\pi} \frac{d x}{4 \pi} \widehat{\Omega}^{(0)}(x, n) f\left(\frac{x}{2}, n \hbar\right) \\
+\sum_{n=-\infty}^{\infty} \int_{\pi}^{2 \pi} \frac{d x}{4 \pi} \widehat{\Omega}^{(0)}(x, n) f\left(\frac{x}{2}, n \hbar\right),
\end{aligned}
$$

using the property (see expression (3.21))

$$
\widehat{\Omega}^{(0)}(x \pm 2 \pi, n)=(-1)^{n} \widehat{\Omega}^{(0)}(x, n),
$$

and the fact that the period of the function $f(x, n \hbar)$ is $2 \pi$, we finally arrive to

$$
\widehat{f}=\sum_{n=-\infty}^{\infty} \int_{-\pi}^{\pi} \frac{d x}{4 \pi} \widehat{\Omega}^{(0)}(x, n)\left(f\left(\frac{x}{2}, n \hbar\right)+(-1)^{n} f\left(\frac{x}{2}+\pi, n \hbar\right)\right) .
$$

This expression (5.5) is equivalent to (3.20) when $k=0, a=2 \pi$ and $F(x, n)$ is given by $(3.17)$.

\subsection{Moyal quantization by WBZ versus Kasperkovitz-Peev formalism}

The quantum formalism introduced by Kasperkovitz and Peev [21] is based on the Weyl quantization.

Let us start from formula (3.18). Taking $k=0$ (i.e. periodic boundary conditions), $a=2 \pi$ and making the variable change $y^{\prime}=y+x / 2$ we obtain formula (110) of [21]

$$
A^{I I}(Q, Y)=\int_{0}^{2 \pi} d y^{\prime} e^{i Q\left(Y-2 y^{\prime}\right)} K_{f}^{(0)}\left(y^{\prime}, Y-y^{\prime}\right),
$$

with the identifications

$$
A^{I I}(Q, Y)=2 \pi F(Y, n), \quad Q=\pi n / a=n / 2 .
$$

Thus, our Weyl symbol (3.17) coincides with the function $A^{I I}$, introduced in [21]. Also $A^{I I}$ verifies a condition analogous to (3.19). It was obtained after cumbersome computations, and it is necessary to put by hand a period of $4 \pi$. However, in our case expressions (3.18) and (3.19) have been easily obtained from the properties of a quantization method well defined for the cylinder like is the correspondence (3.6). Our formalism and the formalism developed by Kasperkovitz and Peev are both based on the Weyl correspondence on $\mathbb{R}^{2}$. In particular, the formal similarity that exists between expressions (2.5) and (3.18) (when $k=0, a=2 \pi$ ) explains the fact that it is possible a construction like has been made in [21]. Moreover, these expressions show that such a comparison can be only made after introducing the double period $4 \pi$.

It is worthy noting that the Weyl correspondence on the cylinder directly distinguishes between the 'classical' phase space $S^{1} \times \mathbb{R}$ and the 'quantum' phase space $S^{1} \times \mathbb{Z}$. The second one is the only one that admits a Moyal formalism based on the 
use of symbols. The correct way of defining these symbols comes from the properties of the Weyl correspondence on the cylinder.

Some features of this formalism that had not been sufficiently explained in the literature appear in our case as simple consequences of the method.

The Moyal quantizer that we have obtained has trace different from one. This fact is related with the use of symbols of functions instead of function themselves. However, with some small modifications our formalism works well and is equivalent to other procedures as we have showed above.

\section{Acknowledgements}

We acknowledge Stephan de Bièvre for his fruitful commentaries. This work has been partially supported by DGES of the Ministerio de Educación y Cultura of Spain under Project PB98-0360, by the DGI of the Ministerio de Ciencia y Tecnología of Spain (project BMF2002-02000) and the Programme FEDER of the European Community, and the Junta de Castilla y León (Spain). J. T. acknowledges la Secretaría de Estado de Educación y Universidades del Ministerio de Educación y Cultura of Spain for the grant (SB2000-0129) that supports his stay in the Universidad de Valladolid.

[1] Moyal J E 1949 Proc. Camb. Phil. Soc. 4599

[2] Weyl H 1931 The theory of groups and quantum mechanics (New York: Dover)

[3] Wigner E P 1932 Phys. Rev. 40749

[4] Groenewold H J 1946 Physica 12405

[5] de Groot S R 1974 La transformation de Weyl et la fonction de Wigner: une forme alternative de la mécanique quantique (Montréal: Les Presses de l'Université de Montréal)

[6] Amiet J P, Huguenin P 1981 Mécaniques classique et quantique dans l'espace de phase (Neuchâtel: Université de Neuchâtel)

[7] Gadella M 1995 Fortschr. Phys. 43229

[8] Berezin F A 1974 Math. URSS Izv. 381116

[9] Berezin F A 1975 Math. URSS Izv. 39 363; 1975 Comm. Math. Phys. 40153

[10] Bayen F, Flato M, Fronsdal C, Lichnerowicz A and Sternheimer D 1978 Ann. Phys. 11161 and 111

[11] Souriau J M 1970 Structure des systèmes dynamiques (Paris: Dunod)

[12] Woodhouse N 1980 Geometric quantization (Oxford: Clarendon Press)

[13] Perelomov A M 1986 Generalized coherent states and their applications (Berlin: Springer)

[14] Weil A 1964 Acta Math. 111143

[15] Brezin J 1970 Trans. Amer. Math. Soc. 150611

[16] Zak J 1967 Phys. Rev. Lett. 191385

[17] Berry M V 1977 Phil. Trans. R. Soc. London A 287237

[18] Mukunda N 1979 Am. J. Phys. 47182

[19] Kasperkovitz P, Foidl C and Dirl R 1987 in The physics of phase space p. 267 (Berlin: Springer)

[20] Arnold A, Degond P, Markowich P A and Steinrück H 1989 Appl. Math. Lett. 2187

[21] Kasperkovitz P and Peev M 1994 Ann. Phys. 23021

[22] Arratia O, Martín M A and del Olmo M A 1996 Rend. Sem. Mat. Univ. Pol. Torino 54191

[23] Arratia O and del Olmo M A 1997 Rep. Math. Phys. 40, 149

[24] Plebański J F, Przanowski M, Tosiek J and Turrubiates f J 2000 Acta Phys. Pol. B 31561

[25] Janssen A J E M 1982 J. Math. Phys. 23720

[26] Folland G B 1989 Harmonic analysis in phase space (Princeton NJ: Princeton Univ. Press) 
[27] Reed M and Simon B 1978 Methods of modern mathematical physics IV: analysis of operators (New York: Academic Press)

[28] Voros A 1977 Dveloppements semi-classiques Ph.D. Thesis Université de Paris-Sud

[29] Voros A 1978 J. Funct. Anal. 29104

[30] J. F. Plebański J F, Przanowski M and Tosiek J 1996 Acta Phys. Pol. B 271961

[31] Grossmann A 1976 Commun. Math. Phys. 48191

[32] Royer A 1977 Phys. Rev. A 15449

[33] Stratonovich R L 1956 Sov. Phys. JETP 311012

[34] de Biévre S and González J A 1993 Semiclassical behaviour of coherent states on the circle Quantization and coherent states methods (Singapore: World Scientific)

[35] González J A 1996 Coherent States and Quantization on the Cylinder Ph. D. Thesis Universidad de Valladolid

[36] Klauder J R and Skagerstam B S 1985 Coherent states: applications in physics and mathematical physics (Singapore: World Scientific)

[37] González J A and del Olmo M A 1998 J. Phys. A 318841

[38] Mumford D 1983 Tata lectures on theta I (Boston: Birkhäuser)

[39] Erdélyi A (ed.) 1981 Higher transcendental functions II, (Malabar FL: Krieger)

[40] Kowalski K, Rembieliński J and Papaloucas L C 1996 J. Phys. A 294149

[41] Kowalski K and Rembieliński J 2002 J. Phys. A 351405

[42] Ali S T, Antoine J P and Gazeau J P 1991 Ann. Inst. H. Poincaré 55829 and 857

[43] Reed M and Simon B 1975 Methods of modern mathematical physics II: Fourier analysis, selfadjointness (New York: Academic Press). 